Mens

revue d'histoire intellectuelle de l'Amérique française

\title{
Une lutte contre " le plus forcené des Yankees » : Edmond de Nevers et les Irlandais des États-Unis
}

\section{Jean-Philippe Warren}

Volume 10, numéro 2, printemps 2010

URI : https://id.erudit.org/iderudit/1023306ar

DOI : https://doi.org/10.7202/1023306ar

Aller au sommaire du numéro

Éditeur(s)

Centre de recherche en civilisation canadienne-française

ISSN

1492-8647 (imprimé)

1927-9299 (numérique)

Découvrir la revue

Citer cet article

Warren, J.-P. (2010). Une lutte contre "le plus forcené des Yankees » : Edmond de Nevers et les Irlandais des États-Unis. Mens, 10(2), 7-47.

https://doi.org/10.7202/1023306ar
Résumé de l'article

À partir d'une discussion portant sur les relations entre les Irlandais et les Canadiens français, cet article présente les grandes lignes de la pensée racialiste d'Edmond de Nevers (1862-1906), un auteur chez qui se lit sans doute le mieux la théorie des races qui dominait chez l'élite intellectuelle à son époque. L'incorporation annoncée des Canadiens français au sein de la grande République américaine représentait, à ses yeux, l'occasion d'une confrontation inévitable avec l'esprit collectif irlandais. Quelles seraient les relations entre ces deux groupes majoritairement catholiques ? Comment allaient-ils interagir ? Et surtout, qu'est-ce que la " science des races ", telle qu'interprétée par Nevers, pouvait bien apprendre à ceux et celles qui tentaient de scruter l'avenir de l'Union américaine à un moment névralgique de son histoire? 


\title{
Une lutte contre « le plus forcené des Yankees" : Edmond de Nevers et les Irlandais des États-Unis ${ }^{1}$
}

\author{
Jean-Philippe Warren \\ Chaire Concordia d'études sur le Québec \\ Département de sociologie et d'anthropologie \\ Université Concordia \\ Il [l'Irlandais] est le plus Américain des \\ Américains, le plus forcené des Yankees, le \\ plus glorieux de l'aigle aux ailes déployées. \\ EdMOND DE NeVERS
}

\section{Résumé}

À partir d'une discussion portant sur les relations entre les Irlandais et les Canadiens français, cet article présente les grandes lignes de la pensée racialiste d'Edmond de Nevers (1862-1906), un auteur chez qui se lit sans doute le mieux la théorie des races qui dominait chez l'élite intellectuelle à son époque. L'incorporation annoncée des Canadiens français au sein de la grande République américaine représentait, à ses yeux, l'occasion d'une confrontation inévitable avec l'esprit collectif irlandais. Quelles seraient les relations entre ces deux groupes majoritairement catholiques? Comment allaient-ils interagir? Et surtout, qu'est-ce que la "science des races ", telle

\footnotetext{
${ }^{1}$ Je tiens à remercier les évaluateurs anonymes de Mens pour leurs conseils et commentaires. Simon Jolivet a bien voulu lire une version préliminaire de ce texte et formuler de précieuses et généreuses critiques.
} 
qu'interprétée par Nevers, pouvait bien apprendre à ceux et celles qui tentaient de scruter l'avenir de l'Union américaine à un moment névralgique de son histoire?

\section{Abstract}

Using a discussion of the relations between the Irish and French $\mathrm{Ca}$ nadians as a starting point, this article presents an overview of the racialist thought of Edmond de Nevers (1862-1906), an author whose work perhaps best expresses the racial theories that prevailed among the intellectual elite of his era. The expected incorporation of French Canada into the American Republic would, in his view, lead to an inevitable confrontation with the collective spirit of the Irish. What sort of relations would these two largely Catholic groups have? How would they interact? And, most significantly, what could people who wished to gaze into the future of the American Union at a vital moment in its history learn from the "science of races," as interpreted by de Nevers.

Les analyses historiques sur le racisme d'avant la Révolution tranquille se sont souvent bornées à souligner l'antisémitisme des uns ou les stéréotypes anti-Noirs ou anti-Amérindiens des autres, se contentant d'évoquer les citations les plus cruelles et les plus bêtes, en constituant une sorte de florilège des préjugés colportés par les Canadiens français ${ }^{2}$. Rares sont les historiens ayant voulu se pencher sur la théorie des races au fondement de telles réflexions xénophobes,

\footnotetext{
${ }^{2}$ L'exemple extrême est sañs doute celui d'Esther Delisle, dont les thèses sur le chanoine Lionel Groulx, publiées dans le contexte du deuxième référendum et des débats autour des notions de nation civique et de nation ethnique, ont provoqué une flambée de réactions dans le milieu universitaire - lire, entre autres, Le traître et le juif: Lionel Groulx, Le Devoir, et le délire du nationalisme d'extrême droite dans la province de Québec (1929-1939), Outremont, L'Étincelle, 1992. Sur l'antisémitisme dans la pensée des élites canadiennes-françaises, qui ne fait pas partie de cet article, lire Gérard Bouchard, «Les rapports avec la communauté juive : un test pour la
} 
sans doute parce qu'ils ne concevaient pas que celles-ci puissent relever d'une idéologie cohérente et complexe. La haine des autres était mentionnée comme un fait brut, quand elle n'était pas aussitôt replacée dans un contexte généralisé de mépris qui n'excluait nulle société. En particulier, la hantise des étrangers chez les écrivains nationalistes du Québec francophone était renvoyée au sentiment similaire qui prévalait alors dans la communauté de langue anglaise. Les sentiments discriminatoires étaient ainsi désamorcés en partie grâce à la méthode comparative, ce qui permettait, du même coup, d'évacuer la nécessité d'en comprendre le sens et la logique ${ }^{3}$.

Dans cet article, je voudrais présenter les grandes lignes de la pensée racialiste ${ }^{4}$ d'Edmond de Nevers (1862-1906), un auteur chez qui se lit sans doute le mieux la théorie des races qui dominait chez l'élite intellectuelle à son époque. Je le ferai à partir d'une discussion de la "question irlandaise " (comprise ici comme celle des relations ethniques, sans référence aucune au débat entourant l'indépendance et la partition de l'Irlande), car c'est aux Irlandais que Nevers a

nation québécoise ", dans Pierre Anctil, Ira Robinson et Gérard Bouchard (dir.), Juifs et Canadiens français dans la société québécoise, Sillery, Septentrion, 2000, p. 13-31.

3 " [...] il faut dégonfler certains mythes qui attribuent aux nationalistes canadiensfrançais le monopole de l'antisémitisme au Canada et qui cherchent ainsi à prouver la nature antidémocratique de la société québécoise. Une étude récente démontre qu'au Canada anglais les antisémites étaient encore plus nombreux et mieux organisés que ceux du Québec et que les milieux outre-Outaouais ont été très complaisants à leur égard " (Denis Monière, Le développement des idéologies au Québec: des origines à nos jours, Montréal, Québec Amérique, 1977, p. 283). Pour un bon exemple récent d'une telle perspective comparatiste, lire Gérard Bouchard, "L'imaginaire de la grande noirceur et de la Révolution tranquille : fictions identitaires et jeux de mémoire au Québec ", Recherches sociographiques, vol. XLVI, n 3 (septembredécembre 2005), p. 411-436.

${ }^{4}$ Racialisme désigne dans ce texte une pensée basée sur une classification théorique de l'espèce humaine en différentes races, caractérisées chacune par des aptitudes et des attitudes " naturellement " distinctes. Sur ce concept, lire Pierre-André Taguieff, La couleur et le sang : doctrines racistes à la française, Paris, Éditions Mille et une nuits, 1998. 
consacré les pages les plus consistantes et les plus éclairantes de son maître ouvrage, L'âme américaine. Il est évident qu'il a aussi discuté, là comme ailleurs dans son œuvre, d'autres nationalités avec lesquelles ses compatriotes sont entrés en contact, que ce soient les Français, les Anglais ou les Allemands 5 . Cependant, plus que toute autre nationalité établie en sol américain, ce sont les Irlandais qui lui offrirent les plus "belles occasions" d'exposer ses théories raciales. Lui qui avait eu des camarades irlandais au collège de Nicolet (qui occupaient ce qu'on appelait alors le " coin des Paddies ") avait été très tôt frappé par les nombreux parallèles que l'on pouvait dresser entre l'Irlande et le Canada français. Or, voilà que, grâce à l'immigration massive de 1820-1860, les histoires de ces deux peuples convergeaient de manière concrète sur le sol du Nouveau Monde. Quelles seraient les relations entre ces deux groupes majoritairement catholiques? Comment allaient-ils interagir? Et surtout, qu'est-ce que la " science des races " pouvait bien apprendre à ceux et celles qui tentaient de scruter l'avenir de l'Union américaine à un moment névralgique de son histoire ${ }^{6}$ ?

Cet article ne s'intéresse pas à la question déjà assez bien balisée des destins parallèles des nations irlandaise et québécoise ${ }^{7}$. Centré plutôt autour de la pensée racialiste d'Edmond de Nevers, cet article est divisé en quatre parties. Dans la première, il présente les grandes lignes de l'utopie en quelque sorte multiculturelle d'Edmond de Nevers, formulée à la toute fin du XIx ${ }^{\mathrm{e}}$ siècle. Dans L'avenir du peuple

\footnotetext{
${ }^{5}$ Lire Edmond de Nevers, La question des races: anthologie, textes choisis et présentés par Jean-Philippe Warren, Montréal, Bibliothèque québécoise, 2003.

${ }^{6}$ Pour un excellent survol récent de l'historiographie portant sur les relations irlandoquébécoises, lire Simon Jolivet, "Survol d'une historiographie irlando-québécoise en développement ", Mens, vol. IX, n 1 (automne 2008), p. 81-115. Consulter aussi Robert Grace, The Irish in Quebec: An Introduction to the Historiography, Montréal, Institut québécois de recherche sur la culture, 1993.

${ }^{7}$ David A. Wilson, "The Irish in North America, New Perspectives ", Acadiensis, vol. XVIII, no 1 (automne 1988), p. 199-215.
} 
canadien-français, celui-ci avait rêvé de voir s'épanouir sous le drapeau étoilé au moins trois grandes communautés ethniques, à savoir les communautés canadienne-française, allemande et anglo-saxonne. Il n'avait pas alors vu se dresser d'obstacle majeur à la réalisation de cette cohabitation pacifique. C'est seulement dans L'âme américaine qu'il prit en considération l'évolution récente des États-Unis à partir d'une grille de lecture racialiste et qu'il s'aperçut de l'importance de considérer de manière propre la question irlandaise, et ce, dans la mesure où les États-Unis s'étaient peu à peu transformés, selon lui, en nation celte. L'incorporation annoncée des Canadiens français au sein de la grande République américaine représentait à ses yeux l'occasion d'une confrontation inévitable avec l'esprit collectif irlandais. Cette partie est donc consacrée à l'exploration de la mutation historique de l'Amérique puritaine en une Amérique celte et aux raisons qui expliquent l'emprise des Irlandais sur ce que Nevers appelait l'âme américaine. La troisième partie décrit les caractéristiques censément innées du tempérament irlandais. Enfin, la dernière partie montre en quoi le caractère irlandais avait, pour Nevers, des conséquences néfastes sur le développement et le rayonnement du groupe canadien-français.

\section{Le rêve d'une amphictyonie continentale}

Une des raisons pour lesquelles les historiens ont eu quelque difficulté à cerner le sens de l'œuvre de Nevers, c'est qu'ils n'ont pas encore assez pris au sérieux la rupture idéologique qui coupe pour ainsi dire sa carrière intellectuelle en deux ${ }^{8}$. On n'a pas compris que Nevers était à sa façon un castor rouge, comme les appelait Wilfrid Laurier, se liant d'amitié avec des gens comme Henri Bourassa et frayant avec les prêtres du Séminaire de Québec. Il faut dire que, aux yeux de ses contemporains eux-mêmes, cet " esprit primesautier " à "l'imagination vagabonde" " projetait l'image d'un homme pétri de

${ }^{8}$ Gérard Bouchard, La pensée impuissante : échecs et mythes nationaux canadiensfrançais (1850-1960), Montréal, Boréal, 2004.

${ }^{9}$ Hormisdas Magnan, "Edmond De Nevers : notice biographique (suite) ", Le Progrès du Saguenay, 26 avril 1906, p. 1. 
contradictions : libre penseur attaché à l'Église romaine, esprit savant qui eut un jour l'idée de devenir cultivateur, il a navigué toute sa vie entre des extrêmes qui paraissent de prime abord irréconciliables. Le lecteur pourra se faire une idée du caractère ambivalent de l'œuvre de Nevers à observer la fureur qui s'abattit de toutes parts sur la dépouille du pauvre homme lorsqu'il mourut en avril 1906. Des journalistes libéraux l'accusèrent d'avoir été un esprit borné et traditionaliste, alors que des critiques religieux dénoncèrent sa malhonnêteté et son impiété ${ }^{10}$. On ne savait trop que faire avec les écrits d'un tel individu, et chacun, semble-t-il, fut soulagé de voir son œuvre tomber rapidement dans l'oubli ${ }^{11}$.

Au moment où Nevers échoue à Paris, en 1890, l'intelligentsia européenne prenait un tournant conservateur qui l'éloignait de l'idée de contrat social et civique et la rapprochait d'une conception holiste de la nation ${ }^{12}$. Plus spécifiquement, Nevers se passionna pour un vaste courant intellectuel, situé entre psychologie collective et anthro-

${ }^{10}$ Dans "La littérature française au pays de Jacques Cartier " (Le Mercure de France, novembre 1906, p. 32), René de Marmande dénonça la présence de l'antidreyfusard Edmond de Nevers aux côtés du consul général de France à Montréal, Alfred Kleczkowski. Olivar Asselin prit la défense de son ami défunt (Le Nationaliste, décembre 1906), ce qui suscita une réplique caustique de la part des directeurs de La Croix ([Anonyme], "À propos d'Edmond de Nevers ", 15 décembre 1906, p. 4).

${ }^{11}$ Un article glissé dans L'Action française en 1919 par Antonio Perrault ("Les précurseurs, Edmond de Nevers ", L'Action française, mai 1919, p. 193-218) fut à peu près tout ce qui se publia sur lui avant 1960 . Depuis, les études sur Edmond de Nevers se sont enrichies de plusieurs articles et ouvrages. Consulter la bibliographie, établie par Jacques Blais, incluse dans Edmond de Nevers, $\grave{A}$ propos de culture intellectuelle, Québec, Nota bene, 2004. Sur la vie et la carrière de Nevers, lire Jean-Philippe Warren, Edmond de Nevers : portrait d'un intellectuel, Montréal, Boréal, 2005. Sur le contexte général de son époque, lire Yvan Lamonde, Histoire sociale des idées au Québec, tome II : 1896-1929, Montréal, Fides, 2004.

${ }^{12}$ Claude Liauzu, La société française face au racisme : de la Révolution à nos jours, Bruxelles, Éditions Complexe, 1999, p. 95. Philippe Claret, La personnalité collective des nations: théories anglo-saxonnes et conceptions françaises du caractère national, Bruxelles, Établissement Émile Bruylant, 1998, p. 90-104. Carole Reynaud-Paligot, La République raciale : paradigme racial et idéologie républicaine (1860-1930), Paris, Presses universitaires de France, 2006. 
pologie raciale, qui connaissait un essor fulgurant en cette fin de siècle, courant intellectuel qui consacrait l'existence d'atavismes collectifs tellement profonds qu'ils confinaient censément à des instincts de race. Se rapprochant de la définition de la nationalité offerte par les ultramontains, pour qui l'affinité nationale découlait d'une essence spirituelle et providentielle manifestée dans l'histoire ${ }^{13}$, Nevers proposera bientôt une conception du peuple fondée sur la voix $d u$ sang et la voix des morts, sans nier pour autant la perfectibilité humaine et l'appel, lointain, d'un certain universel des cultures ${ }^{14}$. Il s'entendait sur ce point avec son ami Henri Bourassa, qui parlait lui aussi de la "voix du sang " qui caractérisait les races humaines au-delà des traits physiques héréditaires et déterminait le caractère national ${ }^{15}$. Tous deux croyaient en la vocation distincte des peuples, qui avaient reçu un legs d'aptitudes physiques, morales et intellectuelles qui correspondait au rôle qu'une certaine Providence leur avait assigné dans l'histoire. En attendant la réconciliation ultime de tous les êtres humains, chaque groupe devait obtenir sa place au soleil des nations.

${ }^{13}$ Gérard Bouchard, "Apogée et déclin de l'idéologie ultramontaine à travers le journal Le Nouveau Monde, 1867-1900 ", dans Fernand Dumont et al. (dir.), Idéologies au Canada français, 1850-1900, Québec, Les Presses de l'Université Laval, 1971, p. 123-124.

${ }^{14}$ Pour un tour d'horizon des termes pays, patrie et nation, ainsi que de leur utilisation au Québec au XIX ${ }^{e}$ siècle, lire John Hare, "Pays, patrie, nation : les liens d'appartenance dans le monde moderne ", Critère, ${ }^{\circ} 22$ (été 1978), p. 211-234. De manière plus générale, lire Damien-Claude Bélanger, "L'antiaméricanisme et l'antimodernisme dans le discours de la droite intellectuelle du Canada, 1891-1945", Revue d'histoire de l'Amérique française, vol. LXI, $\mathrm{n}^{\text {os }}$ 3-4 (hiver-printemps 2008), p. 501-530.

15 " [Henri Bourassa] croyait que chacune des grandes nations de l'Europe possédait une origine commune dans le sang - qu'on était donc latin, anglo-saxon, teuton ou slave. De chaque race jaillissait une combinaison particulière de traits affectifs et mentaux qui déterminaient le caractère national de chaque peuple ou "race". Pour Bourassa, le mot "race" comprenait implicitement des tendances et des instincts de la race " (Joseph Levitt, "La perspective nationaliste d'Henri Bourassa, 1896-1914 ", Revue d'histoire de l'Amérique française, vol. XXII, n 4 (mars 1969), p. 569). Levitt s'appuie, entre autres, sur un texte de juin 1902, paru dans la Revue canadienne (vol. XVI, $\mathrm{n}^{\circ}$ 1), "Le patriotisme canadien-français ". Sur les idées de Bourassa quant à la question irlandaise, lire, de cet auteur, Ireland and Canada: an Address Delivered in Hamilton, Montréal, Imprimerie Le Devoir, 1914. 
Nevers rajoutait toutefois que le métissage et le brassage des peuples ne pouvaient en définitive qu'appauvrir et affaiblir les liens communautaires, ne ratant pas une occasion de dénoncer des emprunts culturels qu'il percevait comme autant de contaminations. Ce qui l'intéressait - comme le titre de son maître ouvrage l'indique -, c'était l'âme des nations, mais une âme qui vibrait d'une longue hérédité et qui s'exprimait à travers le "génie " des nationalités. Pour lui, les traits de caractère des peuples s'enracinaient dans un terreau historique et biologique, suivant en cela la célèbre trilogie de Taine : " la race, le milieu, le moment ${ }^{16}$ ". Les débats sur la définition culturelle ou génétique du mot " race " sont donc assez oiseux dans le cas de Nevers ${ }^{17}$. La race était pour lui une création de l'histoire, certes, mais cette histoire était inscrite de manière si intime dans l'inconscient des individus qu'ils ne pouvaient, malgré de douloureux efforts, échapper tout à fait à ce déterminisme.

Les idées racialistes de Nevers l'avaient convaincu que la société canadienne-française ne serait jamais aussi belle et aussi forte que close sur elle-même. Certes, il approuvait que les élites se mêlent entre elles et goûtent aux plaisirs de l'exotisme, mais ce qui était bon pour les âmes les plus distinguées ne saurait s'appliquer aux esprits les plus frustes et les plus influençables.

En thèse générale, s'il est désirable qu'un cosmopolitisme de bon aloi s'introduise dans les mœurs des classes dirigeantes, et qu'une grande courtoisie préside aux rapports de peuple à peuple, on ne peut nier, au moins d'après les idées qui prévalent encore de notre temps, que l'exclusivisme des masses, leur injustice même, leur fanatisme souvent, ne soient une force pour une nation

${ }^{16}$ Claret, La personnalité collective des nations.

${ }^{17}$ Voir les pages éclairantes de Julien Goyette sur les notions de nation, de nationalité, de peuple, de race, de communauté culturelle et d'ethnie chez Groulx - Lionel Groulx: une anthologie, textes choisis et présentés par Julien Goyette, Montréal, Bibliothèque québécoise, 1998, p. 71-73 et 75-80. Lire aussi Pierre Hébert, Lionel Groulx et l'appel de la race, avec la collaboration de Marie-Pier Luneau, Montréal, Fides, 1996. 
composée d'éléments homogènes, et dont l'union est l'œuvre de plusieurs siècles ${ }^{18}$.

Nevers offrait en modèle un jeune élève d'une école secondaire américaine qui, à toutes les questions posées sur les plus illustres représentants des arts et de la science, donnait pour réponse des noms de célébrités anglophones. Le plus grand musicien, ou le plus grand général, ou le plus grand artiste, n'aurait pu, pour ce jeune garçon élevé dans le chauvinisme national, appartenir à toute autre nation que la sienne. Nevers admirait une telle attitude. Il souhaitait que le jeune Canadien français reçoive une éducation aussi bornée et exclusive, où les Bossuet, les Delacroix, les Victor Hugo et les Napoléon prennent toute la place, sans égard pour les génies des autres peuples.

Dans L'avenir du peuple canadien-français, publié en 1896, Edmond de Nevers paraissait un homme confiant, convaincu que rien ne s'opposait à l'exploitation de ce chauvinisme canadien-français. Même l'annexion du Canada aux États-Unis, qui, selon lui, se préparait inévitablement, ne parviendrait pas à briser les liens sacrés de la nation ${ }^{19}$. L'exode massif des siens, qu'il avait dénoncé avant son voyage aux États-Unis en 1892, lui semblait désormais une promesse extraordinaire. C'est que la naturalisation des Canadiens français, c'est-à-dire l'acceptation de la citoyenneté américaine, allait de pair, chez Nevers, avec une fidélité à son lieu d'origine. Il ne concevait pas de quitter sa patrie en se rendant aux États-Unis, la nationalité étant pour lui affaire non pas d'État, mais de cœur et de sang. Il trouvait des oreilles favorables à ses prêches. Le slogan "Soyons Canadiens avant tout " était entonné par la vaste majorité des élites franco-

\footnotetext{
${ }^{18}$ Edmond de Nevers, L'avenir du peuple canadien-français, Montréal, Fides, 1964, p. 174-175.

${ }_{19}$ Nevers n'est, bien entendu, pas seul à prédire la réunification des Canadiens français de part et d'autre de la frontière américaine. Édouard Hamon, entre autres, contemplait cet avenir avec optimisme dans Les Canadiens français de la Nouvelle-Angleterre (Québec, N-S Hardy, 1891, p. 145-146).
} 
américaines, pour qui il s'agissait de bâtir autour des communautés francophones " une muraille invisible ", " une forteresse morale " en mesure de repousser les assauts de l'anglicisation.

Les intellectuels parisiens étant largement convaincus que l'avenir de l'Europe allait tourner autour de ces puissants pôles d'attraction qu'étaient l'Allemagne, la France et l'Angleterre, Nevers s'imaginait que l'avenir du continent américain était entre les mains des immigrants allemands, anglais et français. Il lui paraissait souhaitable que, un peu comme en Suisse ${ }^{20}$, se constituent dans une Amérique du Nord politiquement unifiée trois foyers nationaux : les Allemands dans l'Ouest, les Canadiens français au Québec et en NouvelleAngleterre, avec quelques îlots dispersés sur le reste du territoire, et les Anglophones partout ailleurs. Les groupes d'origine teutonne, devenus " indomptables et pleins d'espoir dans les destinées de leur race ", allaient préserver en Amérique leur langue et leur culture. Et cela était bien, car ils pourraient alors perpétuer dans une contrée nouvelle les vertus ayant fait la grandeur de leur pays d'origine et " édifier à la science et à l'art les monuments qui sont la gloire la plus pure de la patrie de Goethe et de Wagner ${ }^{21}$ ». Dans l'Ouest se constituerait une Petite Allemagne, avec ses cafés, ses Bier Garten, ses salles de concert, ses écoles, ses journaux, ses églises et ses cercles d'étude. Dans l'Est, de la Nouvelle-Orléans à Montréal, les Canadiens français allaient créer sur le même modèle une France d'outre-mer (Nevers songeait à une seconde édition de L'avenir du peuple canadienfrançais qui aurait porté le titre France d'Amérique). Les Suédois et les Polonais allaient se constituer à leur tour en communautés distinctes. Il n'était pas impossible que les Irlandais ne cèdent au

${ }^{20}$ Edmond de Nevers, "L'évolution des peuples anciens et modernes ", Revue canadienne, tome XLVII (septembre 1904), p. 284. Edmond de Nevers, "Encore quelques mots sur les Anglais et nous ", Le Soleil, 8 mars 1902, p. 1. D'autres auteurs canadiens prenaient la Suisse pour modèle, tel F. R. LeRoux, "Numéro souvenir ", Le National (Lowell, MA), juillet 1892.

${ }^{21}$ Edmond de Nevers, L'áme américaine, tome II : L'évolution - À travers la vie américaine - Vers l'avenir, Paris, Jouve \& Royer, 1900, p. 359. 
mouvement et ressuscitent le gaélique - L. I. Boivin prédisant la constitution d'une Verte-Irlande dans le Sud du pays à côté d'une Jeune-Allemagne dans l'Ouest et d'une Nouvelle-France dans l'Est ${ }^{22}$. C'est ainsi que les États-Unis formeraient dans l'avenir une immense amphictyonie continentale, à savoir une association de nations placées sous le patronage d'un président distant et sans autre pouvoir réel que celui de défendre les frontières politiques, et montreraient à la face du monde comment de petites patries - "Petits Canadas", "Petites Allemagnes ", "Petites Angleterres » - pouvaient cohabiter au sein de la "grande patrie".

Dans les centres dynamiques de Lowell, de Central Falls ou de Fall River, les masses de gens parlant français n'auraient pas à s'inquiéter des abus du pouvoir central. Protégés par les traités, reconnus pour la douceur de leurs mœurs, contribuant comme les autres à la prospérité de l'Union, les Canadiens français ne devaient craindre nul ennemi. D'ailleurs, les rares vexations dont ils pourraient être victimes dans des États-Unis élargis n'avaient rien pour effaroucher Nevers : devant ces frustrations d'amour-propre, l'ardeur nationale de ses compatriotes promettait de n'en être que plus forte. Elles fouetteraient chez eux la vieille combativité gauloise! De telles manifestations de mépris de la part des autres peuples qui habitaient le continent paraissaient, de toute façon, fort improbables. Pourquoi, demandait Nevers en 1896, les descendants d'Italiens, de Slaves, de Hongrois ou de Scandinaves voudraient-ils bafouer les droits des Canadiens français de parler leur langue et de pratiquer leur religion, eux qui, souvent, ont versé de chaudes larmes sur leurs traditions perdues au moment où la guerre et la famine les forçaient à émigrer? Qu'est-ce qui pourrait bien pousser des nouveaux arrivants ayant souffert l'oppression à vouloir imposer à d'autres la pire des injustices? Non, pour Nevers, une telle aberration ne saurait être. De ce côté-là,

${ }^{22}$ L. I. Boivin, "L'avenir de notre race ", Revue nationale, article reproduit dans L'Opinion publique, 17 mai 1895, p. 6. 
les Canadiens français qui migraient vers le sud n'avaient, semblaitil, rien à craindre.

Une seule ombre demeurait au tableau quand Edmond de Nevers s'était enfermé dans les bibliothèques de Londres pour écrire, en trois mois fiévreux, l'essentiel de L'avenir du peuple canadien-français: les Irlandais. Dans une petite note glissée en fin de volume, Nevers soulignait que, par un "fait étrange ", " les principaux champions de l'anglicisation sont des Irlandais ». Il semblait même, selon ce qu'il entendait des événements outre-Atlantique, que l'évêque de SaintPaul de 1884 à 1918, M ${ }^{\mathrm{gr}}$ John Ireland lui-même, aurait été le porte-drapeau de l'imposition de la langue anglaise sur le nouveau continent ${ }^{23}$. Ce fait semblait troubler Nevers. Il paraît hésitant de reconnaître de tels comportements, ayant visiblement quelque peine à croire que les Irlandais, qui avaient subi tant d'abus de la part des conquérants anglais, puissent se faire les alliés de leurs anciens oppresseurs. Le temps passé aux États-Unis allait vite le faire déchanter. Le curé Jean-Frédéric Audet ne déclarait-il pas que l'hostilité entre les Irlandais et les Canadiens français catholiques atteignait de tels sommets, en Nouvelle-Angleterre, que Dieu serait contraint de les séparer au ciel ${ }^{24}$ ! À Manchester (Massachusetts), par exemple, les ouvriers qui traversaient la " commune d'Irlande " devaient affronter non seulement les quolibets mais aussi les coups. « Nous ne pouvions le faire sans que nos vêtements du dimanche ne soient abîmés par les ordures qu'on nous lançait. On nous jetait aussi des pierres. Plusieurs d'entre nous ont reçu de dures raclées de la part de jeunes Irlando-Américains armés de bâtons ${ }^{25}$. "Comment de tels gestes

\footnotetext{
${ }^{23}$ Nevers, L'avenir du peuple canadien-français, p. 214.

${ }^{24}$ Jean Frédéric Audet, Histoire de la Congrégation canadienne de Winooski au Vermont, Montréal, Imprimerie de l'Institution des Sourds-Muets, 1906, p. 41, cité dans Mason Wade, "The French Parish and Survivance in Nineteenth Century New England", The Catholic Historical Review, vol. XXXVI, n 2 (juillet 1950), p. 168.

${ }^{25}$ "We couln't pass there without having our Sunday clothes ruined by filthy swill thrown at us from yards and alleys. Rock flew also, and many of us youngsters received painful beatings from young Irish Americans who were nearly always armed with sticks " (Cité dans C. Stewart Doty (dir.), The First Franco-Americans: New England Life Histories
} 
étaient-ils possibles de la part de coreligionnaires qui partageaient, de plus, un même ennemi historique, l'Angleterre?

\section{Les États (celtes)-Unis d'Amérique}

Quand paraît en 1900 L'âme américaine, le second ouvrage de Nevers, ce dernier a développé plus avant ses réflexions sociologiques. Il a compris désormais l'importance de considérer la question irlandaise de manière plus fine et plus spécifique. Car, des divers facteurs qui expliquaient pour Nevers la transformation de la psychologie collective en Amérique depuis un demi-siècle, nul ne lui semblait plus important que l'influence de l'immigration irlandaise. Comment reconnaître, demandait-il, le puritain réservé et austère dans la population des villes américaines, chicaneuse, tapageuse, agitée et turbulente de la fin du XIX ${ }^{\mathrm{e}}$ siècle? Comment ne pas admettre la distance énorme qui séparait désormais les habitants des hameaux et des villages de la Nouvelle-Angleterre (ces gens à «l'air anguleux ", à « la voix un peu sacerdotale ", ayant "conservé le pli rigide des lèvres, le sérieux impassible de l'expression, le maintien sévère ${ }^{26} "$ ) des habitants exubérants des grands centres? Non, quelque chose s'était passé quelque part entre le XVIII et le XIX ${ }^{e}$ siècle, période pendant laquelle l'ancien esprit national puritain avait été déclassé par un tempérament nouveau, très différent de l'ancien.

Pour comprendre ce changement, Nevers évoquait l'influence de plus en plus prépondérante de la mentalité irlandaise, laquelle avait fait perdre à l'âme américaine ses caractéristiques anglo-saxonnes d'origine. Non seulement " une Irlande nouvelle s'[était] constituée en Amérique ${ }^{27}$ ", mais l'Amérique elle-même s'était en quelque sorte

from the Federal Writers' Project, 1938-1939, Orono, The University of Maine at Orono Press, 1985, p. 31-32). Sur les tensions qui existent au même moment au Québec, lire John Willis, "Le Québec, l'Irlande et les migrations de la grande famine : origine, contexte et dénouement ", dans Marcel Bellavance (dir.), La grande mouvance, Sillery, Septentrion, 1990, p. 115-145.

${ }^{26}$ Nevers, L'àme américaine, tome II, p. 62-63.

${ }^{27}$ Ibid., p. 57. 
hibernisée en subissant la sourde influence celtique. Aux yeux de Nevers, en effet, nulle autre nationalité n'avait réussi à imprimer sa marque sur l'âme ou le génie du peuple américain et à dominer à ce point les us et les mœurs. Le mélange de l'hérédité celtique et de l'hérédité anglo-saxonne avait fait perdre peu à peu à l'âme américaine son austérité et sa retenue légendaires et avait produit un être complexe, à la fois pressé de s'enrichir et consommateur, optimiste et calculateur, âpre au gain et susceptible de succomber à des emballements soudains, décidé et versatile. L'Irlandais avait beau prononcer désormais l'anglais avec un accent nasal, il avait beau avoir dissimulé ses origines sous un chauvinisme américain de bon aloi, il avait conservé les qualités de sa nationalité. " [...] il n'est pas assimilé, il assimile, il reste luimême $e^{28}$. "Surtout - et c'est ici le point capital -, cette combinaison avait renforcé la tendance à mépriser l'étranger, qui est la seule qualité citée par Nevers partagée par les peuples celte et anglo-saxon. Cette xénophobie instinctive explique pourquoi, selon Nevers, les ÉtatsUnis, autrefois nation tranquille et pacifique, avaient pu choisir de suivre la voie tracée par l'Angleterre et se lancer dans des entreprises impérialistes. L'« atavisme inconscient » des Irlandais se réveillait en eux maintenant que les immigrants de la verte Érin étaient libres d'exprimer leurs hérédités dans leur société d'accueil.

[...] l'homme qui incarne en lui l'âme américaine, telle au moins qu'elle se manifeste aux yeux de l'étranger, c'est le Celte. La partie la plus remuante, la plus entreprenante de la population, celle qui conduit les élections, qui pérore, qui brille à la tribune et au barreau, qui boxe, qui établit des championnats, celle qui proclame, sur tous les tons, le nec plus ultra de l'excellence américaine, celle qui s'émeut à tout propos, qui a déclaré la guerre à l'Espagne et occupé les Philippines, cette population a du sang celtique dans les veines ${ }^{29}$.

${ }^{28}$ Ibid., p. 88.

${ }^{29}$ Ibid., p. 60-61. 
Comment les Irlandais, pour la plupart catholiques ${ }^{30}$, avaient-ils réussi à supplanter la tradition puritaine et à imposer leur propre tempérament à la civilisation américaine? Qu'est-ce qui pouvait expliquer cette étrange et profonde transformation des mœurs dans les cinquante dernières années du XIX ${ }^{e}$ siècle? Nevers voyait l'action de quatre causes distinctes dans cette capacité des émigrants irlandais à modifier les traits des autres races - et en particulier ceux de la race anglo-saxonne - qui formaient le formidable melting pot américain.

En premier lieu, du strict point de vue démographique, il aurait été étonnant qu'il en soit autrement. Les calculs rapportés par Nevers sont à première vue probants : sur les 80 millions d'habitants que comptaient les États-Unis, 26 millions auraient été d'origine irlandaise, 20 millions d'origine allemande, 10 à 12 millions d'origine africaine, 1 à 1,5 million d'origine canadienne-française, ce qui laissait, par soustraction, un total de 25 ou 30 millions d'origine anglo-saxonne. Mais ces données étaient incomplètes, car il n'était pas question des descendants de Polonais, de Suédois, de Hollandais, et de tant d'autres pays encore ayant contribué à la société américaine (Nevers faisait état de deux millions et demi de Suédois et de Norvégiens, d'un million et demi d'Italiens, de 600000 Hongrois, etc.). Ces derniers mis dans la balance, les descendants de puritains et d'Anglo-Saxons de vieille souche américaine auraient représenté au plus 6 millions de citoyens, c'est-à-dire moins de $10 \%$ de la population totale vivant sous le drapeau étoilé de l'Union. Par comparaison, les citoyens irlandais, à eux seuls, auraient compté pour au moins le quadruple ${ }^{31}$, dépassant en proportion toute autre nationalité.

${ }^{30}$ Je n'ai pas établi ici une distinction entre Irlandais protestants et Irlandais catholiques, puisque Nevers passe outre cette différence pourtant capitale. Alors que, grosso modo, le Québec a surtout accueilli des Irlandais catholiques, l'Ontario a surtout accueilli des Irlandais protestants, plus enclins à soutenir les vues et les idéaux britanniques. Aux États-Unis, une majorité d'immigrants irlandais étaient catholiques. Lire Jolivet, "Survol d'une historiographie irlando-québécoise en développement ", p. 90.

${ }^{31}$ Voir les calculs d'Edmond de Nevers dans L'àme américaine, tome I : Les origines - La vie historique, Paris, Jouve \& Royer, 1900, p. 341-342. Ce tableau est approximatif, et 
La seule explication démographique semblait toutefois incomplète. Nevers croyait aussi que l'Amérique avait spontanément adopté le caractère de l'Irlandais parce que l'Anglais avait reconnu en lui un frère sous la carapace de retenue et de moralité que lui avait imposée la Réforme protestante. Pour Nevers, les vrais Anglais, les Anglais des temps anciens, ceux du temps de la reine Anne, les Anglais de la Merry England, étaient des gens gais et colorés. "C'est la religion réformée qui leur a donné la physionomie particulière qui est devenue la leur; lorsque cette religion a sombré ils ont pu facilement reprendre, au contact du Celte, leur âme d'autrefois ${ }^{32}$. "Le côté policé, hautain et superbe de l'habitant de la Nouvelle-Angleterre n'était finalement qu'une façade, derrière laquelle se cachait un Celte enjoué et déluré. En ébranlant les bases de la spiritualité protestante, l'histoire américaine avait facilité la libération de la personnalité enfouie des Anglais.

Ensuite, Nevers mentionnait le fait que la société démocratique favorisait par sa dynamique propre l'éclosion de manifestations bruyantes et populaires, comme le charlatanisme. Aussi, les tendances naturelles à l'exagération des Irlandais y auraient trouvé un terreau fertile. Le citoyen moyen des États-Unis décrit par Nevers paraît le plus souvent grossier, affairé, pratique, roublard, exubérant, bouffon ; il correspond assez bien au portrait de l'Irlandais typique brossé par Nevers ${ }^{33}$ (lequel, sans s'en apercevoir, recyclait dans sa rhétorique les arguments des nativistes américains, qui traitaient les Irlandais de violents, d'alcooliques, de grégaires et tutti quanti). L'Irlandais aurait

certaines données compilées par Nevers ne concordent pas avec celui-ci. L'auteur ne vise qu'à donner un aperçu général de la situation, et non pas à parvenir à l'exactitude démographique, qui, en ces matières, est évidemment impossible. J'ai cité ici les "statistiques irrécusables " données dans la préface des Études sur les États-Unis, Québec, Dussault et Proulx, 1902, p. Ix.

${ }^{32}$ Nevers, L'âme américaine, tome I, p. 62-63.

${ }^{33}$ À noter que la théorie des races de Nevers l'empêche de concevoir clairement l'influence du progrès de la démocratie sur les mœurs, comme l'a fait Alexis de Tocqueville. Son explication demeure tout à la fois, comme le soulignait Ferdinand Brunetière, "matérialiste et mystique " ( L'âme américaine ", Revue des deux mondes, tome CLXII (1900), p. 689). 
spontanément embrassé les mœurs démocratiques parce qu'elles étaient déjà en partie les siennes et qu'il pouvait grâce à elles donner libre cours à ses emportements et à ses enthousiasmes. Après des siècles de rage contenue sous le joug de la couronne britannique, il pouvait être en Amérique le sportif le plus exubérant et le plus vantard, le parvenu le plus tape-à-l'œil, le marchand le plus criard. Il était fait pour vivre dans une civilisation tapageuse, enfiévrée, triviale et tourbillonnante. La surexcitation de la vie démocratique s'accorderait parfaitement avec son sang bouillant.

Enfin, aux yeux de Nevers, l'attirance des masses américaines pour l'Irlandais était tout simplement naturelle, compte tenu de son caractère affable et amène. Le vieil habitant de la Nouvelle-Angleterre n'avait su séduire personne par sa froideur et son mépris. Le "Sauvage " l'avait fui, le "Nègre " l'avait haï, l'immigré l'avait évité (je reprends ici le vocabulaire de Nevers). L'Irlandais avait réussi là où le puritain avait échoué en usant de son attitude débonnaire. "Il est certain que l'homme à l'esprit primesautier, à l'imagination féconde, aux impulsions chaleureuses, aux enthousiasmes vibrants possède une plus grande force d'attraction que le taciturne et l'austère, surtout lorsqu'il est le nombre ${ }^{34}$. " L'Irlandais avait fait plus par la bonne humeur pour se rallier les masses américaines que le puritain par la raison et la discipline.

Bref, parce qu'il formait la majorité immigrante, parce que ses traits se rapprochaient de ceux des sociétés démocratiques et, par-delà les siècles, des premiers occupants européens, et parce que son moral s'accordait mieux avec celui des nouveaux arrivants, l'Irlandais ne perdit pas de temps pour dominer les anciennes colonies britanniques. Nevers pouvait par conséquent prétendre que la compréhension de l'évolution de la société américaine ne saurait être complète sans prendre en compte dans l'analyse sociologique la présence irlandaise. En particulier, il se demandait quelles pouvaient être les conséquences de cette dérive identitaire sur l'avenir du peuple canadien-français.

\footnotetext{
${ }^{34}$ Nevers, L'àme américaine, tome II, p. 62-63.
} 
En quoi le fait que les États-Unis formaient un peuple celte dérangeraitil les plans des nationalistes canadiens-français qui s'activaient, en 1900, à préserver les communautés francophones de la NouvelleAngleterre? Les 600 pages de L'âme américaine ont été d'abord écrites pour répondre à cette question : la race canadienne-française sauraitelle trouver sous le drapeau étoilé la sécurité et la reconnaissance de ses droits, qu'elle recherchait ardemment depuis la Conquête, alors même qu'elle devrait côtoyer après la dissolution du Canada d'autres races, peut-être plus farouches que la race anglaise? En d'autres termes, la République américaine se ferait-elle l'alliée ou l'ennemie des descendants des colons de la Nouvelle-France? La race irlandaise, désormais prépondérante aux États-Unis, allait-elle se montrer magnanime? Était-il naturel pour elle, ou au moins pour ses élites, de faire preuve de fair-play? Pour répondre à de telles questions, encore fallait-il bien saisir la nature profonde et les tendances supposément innées du tempérament irlandais.

\section{Le tempérament irlandais}

Au dire de Nevers, la race irlandaise qui prenait pied en Amérique à son époque possédait, comme l'ensemble des races humaines, des qualités et des défauts innés. Chaque nationalité, selon Nevers, charrie des traits spécifiques qui s'imposent aux individus qui en sont membres. Or, ce qui captivait et inquiétait en particulier Nevers, c'est que, parmi les facultés distinctives de la race irlandaise, on ne puisse pas reconnaître la tolérance et le respect de la différence. Selon lui, la race irlandaise serait bien au contraire singulièrement portée à la répression et à l'oppression des autres nationalités. Un journaliste de L'Opinion publique faisait, dans L'Avenir national de 1898, un procès sans nuances du rôle des Irlandais dans l'histoire de la persécution dont étaient victimes les autres groupes ethniques. Là où l'Irlandais peut compter sur le nombre, écrivait-il, «c'est la Terreur érigée en permanence. [...] La vérité est que, quoi qu'il fasse, l'Irlandais est né dominateur, turbulent, et mauvais coucheur. [...] Sa véritable 
nature est, depuis longtemps, l'anglo-saxonisme ${ }^{35}$ ". Prenons garde de croire que de tels commentaires soient l'exclusive des journalistes canadiens-français; les groupes catholiques nationaux qui cherchaient à conserver, qui la langue allemande, qui la langue polonaise, qui la langue hongroise, se butaient eux aussi à la « fièvre assimilatrice " des Irlandais, qui cherchaient à «tout américaniser ".

Cela avait de quoi étonner un homme comme Nevers, qui se piquait de connaitre l'histoire européenne. Les descendants d'Irlandais installés en Amérique n'auraient-ils pas dû comprendre plus que tout autre peuple l'importance de respecter la diversité culturelle, eux qui avaient subi le joug de fer des armées britanniques? Après avoir été "l'éternel opprimé, l'éternel martyr ", après avoir souffert " pendant des siècles, sous une tyrannie dont le monde civilisé n'a jamais encore donné d'exemples ${ }^{36}$ ", n'auraient-ils pas dû réaliser l'iniquité des droits bafoués et des traditions foulées au pied? Les campagnes affamées, les paysans transformés en serfs, la langue réprimée, la religion combattue, les villages pillés, tout cela n'aurait-il pas dû leur donner à jamais le dégoût des préjugés et de l'ostracisme? N'aurait-on pas dû attendre de ces gens, que Nevers avait appris à admirer à travers la lecture des discours patriotiques, une attitude franche et loyale devant les conditions injustes des plus faibles? Surtout, n'auraient-ils pas été mieux placés que quiconque pour comprendre la nécessité de se ranger du côté des minorités méprisées, eux qui étaient assimilés par maints Anglo-Saxons, tout comme les Canadiens français, à de dangereux papistes $^{37}$ ?

35 [Anonyme], "Une nouvelle forme de l'Anglo-Saxonisme ", L'Avenir national, 5 avril 1898 , p. 2.

${ }^{36}$ Nevers, L'âme américaine, tome II, p. 55.

${ }^{37}$ " Il n'est pas impossible, s'inquiétait le maire Matthews, de Boston, qu'avant cinquante autres années la terre des puritains soit occupée en grande partie par les descendants de l'Irlande et de la France " (Cité dans Robert Rumilly, Histoire des Franco-Américains, Montréal, L'Union Saint-Jean-Baptiste d'Amérique, 1958, p. 133). 
Or, plutôt que de se reconnaître solidaire de toutes les victimes de persécution, les émigrés irlandais auraient pris farouchement parti pour l'élément dominant de la société américaine. Habitant avec ses parents à Central Falls dans les années 1890, Nevers croyait remarquer la rage singulière avec laquelle ils persécutaient les groupes migrants qui souhaitaient préserver leurs traditions. "L'Anglo-Saxon, qui se complaît dans la pensée d'une Grande-Bretagne embrassant peu à peu le monde entier, devrait être étonné, s'il pouvait s'étonner, de trouver les plus ardents champions de l'œuvre de l'assimilation anglaise, parmi ceux qu'il a battus, ruinés et ridiculisés ${ }^{38}$." Selon Nevers, les Irlandais seraient devenus "les plus forcenés des Yankees ", déversant sur l'ensemble des foreigners des flots de préjugés et de mépris.

Dans l'agitation "américaine-native" qui, pendant vingt-cinq ans, a concentré en elle presque toute la vie politique du pays, les fanatiques anglo-saxons ont eu avec eux, plus ardents, plus agressifs qu'eux, tous ceux que l'oppression, l'intolérance et le mépris avaient gagnés à leur cause, les Irlandais et descendants d'Irlandais non catholiques $[\ldots]^{39}$.

Alors que les Canadiens de langue française pouvaient prendre fait et cause pour les Irlandais en Europe, il était clair, en règle générale, qu'ils s'opposaient aux immigrants irlandais en Amérique, surtout quand ceux-ci avaient abjuré leurs traditions. L'élite des vrais Irlandais, patriotes, soucieux de leurs racines, fils de Daniel O'Connell et de Thomas More, ne devait pas être confondue avec la foule des faux Irlandais « anglicisés jusqu’à la moelle ${ }^{40}$ ".

${ }^{38}$ Nevers, L'âme américaine, tome II, p. 60.

${ }^{39}$ Ibid., p. 24. Cette opinion était corroborée par James Bryce, The American Commonwealth, London, McMillan and Co., 1890, p. 358.

40 "L'Irlande a perdu sa langue qui est en train de renaître, et quelques-uns de ses fils anglicisés jusqu'à la moelle, sont tellement abâtardis et tellement dénués de toute fierté qu'esclaves ils se font les propagateurs de l'esclavage, et se font les complices des proscripteurs de la langue de la France... " (La Bataille, 9 novembre 1916, p. 1, cité dans Simon Jolivet, Les deux questions irlandaises du Québec, 1898-1921: des considérations canadiennes-françaises et irlando-catholiques, thèse de doctorat, Université Concordia, 2008, p. 141). 
Pour atteindre leur but, ils [les Irlandais] vont jusqu'à rechercher l'alliance des fanatiques protestants; ils soulèvent les préjugés nationaux des Américains contre les étrangers, eux qui sont pourtant si peu Américains qu'ils forment en ce pays une vaste agglomération distincte, unie dans l'égoïsme et dans la haine de la liberté bien entendue, dont le but principal est le triomphe définitif de l'idée irlandaise ${ }^{41}$ !

Ces Irlandais américains se seraient posés en ennemis féroces des groupes qui cherchaient à perpétuer un idiome différent et auraient combattu avec acharnement les velléités de transmettre la langue maternelle aux enfants d'immigrants. "On serait naturellement porté à croire ", déclarait un Irlandais du nom de M. T. Donnell cité par Nevers, "que pour nous venger de l'extirpation de la langue de nos pères, nous emploierions dans l'Église et dans l'État, aussi bien que dans la vie privée, notre influence à combattre la langue de nos ennemis; mais c'est le contraire qui arrive; nous travaillons à la propager, nous voulons même l'imposer aux autres par la violence ${ }^{42}$. " Cela, en soi, faisait fulminer Nevers, qui ne concevait pas qu'une politique aussi funeste puisse être l'objectif d'un peuple moderne, mais il s'empourprait davantage encore devant les tentatives d'interdire l'enseignement de la langue de la plus fidèle alliée de l'Irlande et des États-Unis, la France. Comment donc les Irlandais, demandait-il, pouvaient-ils poursuivre en sol américain les visées assimilatrices de la puissance britannique contre des gens qui parlaient la langue d'une nation amie? Ils auraient dû la chérir, la protéger, la défendre au besoin contre ses détracteurs et ses calomniateurs, au lieu d'en faire l'objet d'un incompréhensible acharnement. En tentant partout de proscrire le français, ils se faisaient, par jalousie et égoïsme, les

${ }^{41}$ [Anonyme], "Irlandais et Canadiens français (suite et fin) ", L'espérance (Central Falls), 9 juin 1891, p. 2.

${ }^{42}$ Nevers ne cite pas l'article dans sa version originale - The Irish Republic (New York), 12 décembre 1897 -, mais à partir d'une transcription de L'Indépendant de Fall River (Nevers, L'âme américaine, tome II, p. 180-181). 
complices d'un crime dont ils avaient les premiers souffert ${ }^{43}$. Ces derniers n'avaient en effet pas d'autre priorité, semblait-il, que de faire partager aux autres groupes leur apostasie nationale en leur faisant abjurer leur langue maternelle. Ils cherchaient par tous les moyens à " anglifier ou irlandiser ${ }^{44}$ » de force les nouveaux arrivants ${ }^{45}$. "[...] parlant aujourd'hui la langue de leur ennemi séculaire, une langue qu'ils ont maudite des milliers de fois, une langue qui ne leur rappelle que misères et persécutions, ils veulent vous faire partager leur ignominie, vous faire abandonner l'usage du français, comme ils ont abandonné celui du gaëlique ${ }^{46}$. " En particulier, les Canadiens français de la Nouvelle-Angleterre étaient confrontés à la morgue de leurs coreligionnaires irlandais, le clergé irlandais, véritable " hibernachie ${ }^{47}$ ", s'étant ligué contre eux pour leur faire renier leur langue.

Les évêques irlandais, au moins la plupart d'entre eux, font tout ce qui est en leur pouvoir pour imposer aux catholiques américains la langue anglaise, ce " canal du protestantisme »; ils combattent, avec un fanatisme aussi aveugle qu'il semble illogique, l'expansion

43 "En effet, il est assez humiliant de convenir entre eux que leur race a abandonné la langue de ses pères pour apprendre l'idiome anglais, qui a servi à faire répandre tant de sang irlandais : il est humiliant, disons-nous, de s'avouer in petto qu'on parle la langue de Cromwell et des bourreaux de Robert Emmet, faute de savoir la langue gaélique, tandis que cette poignée de Canadiens français héroïques abandonnés par la France, au siècle dernier, ont conservé leur idiome maternel au milieu des plus graves périls! L'égoïsme et la jalousie des Irlandais a [sic] créé entre eux et nous un abîme qui ne peut être comblé! " ([Anonyme], "Irlandais et Canadiens français ", p. 2.)

${ }^{44}$ Narcisse-Rodolphe Martineau à M ${ }^{\mathrm{gr}}$ Jacobini, 8 septembre 1885, cité dans Yves Roby, Les Franco-Américains de la Nouvelle-Angleterre, 1776-1930, Sillery, Septentrion, 1990 , p. 121.

45 Jay P. Dolan, The American Catholic Experience: A History from Colonial Times to the Present, Garden City (NY), Doubleday and Co, 1985, p. 180.

${ }^{46}$ L'Avenir national (Manchester, NH), 28 février 1898, cité dans Nevers, L'âme américaine, tome II, p. 181-182.

${ }^{47}$ James Hennesey, American Catholics: A History of the Roman Catholic Community in the United States, Oxford, Oxford University Press, 1981. 
de la langue française dans l'Est, celle des langues allemande et polonaise dans l'Ouest et ne donnent aux paroisses non irlandaises des curés appartenant à la nationalité de ceux qui les composent, que lorsqu'ils y sont contraints ${ }^{48}$.

Il est vrai que la fin du siècle a vu le déclin d'une phase plus modérée de nationalisme irlando-américain et l'éveil d'une attitude beaucoup plus agressive envers les autres communautés ethniques des États-Unis. "Ultimately, in accommodations' place a new Irish ethnic assertiveness, even belligerence, reigned, accompanied by a selfconscious revival of interest in Irish culture ${ }^{49}$. » Quelles étaient les raisons d'un tel zèle assimilateur? Qu'est-ce qui poussait donc les Irlandais à s'acharner, entre autres, sur le peuple canadien-français? Nevers ne voulait pas considérer dans son interprétation globale la dépression économique, le déferlement de vagues migrantes et le retour du nativisme anglo-saxon. Pourtant, nous savons que la crise ayant commencé à sévir dans les années 1890 en Nouvelle-Angleterre a eu des répercussions directes sur les tensions ethniques en dressant les communautés culturelles les unes contre les autres, dans une escalade qui aboutissait souvent à se choisir des boucs émissaires parmi les Italiens, les Polonais ou les Canadiens français. La concurrence sur le marché du travail envenimait des incompréhensions alimentées déjà par la ghettoïsation croissante de la société américaine. Nevers ne mentionnait pas les conflits ouvriers ayant envenimé les relations entre les Irlandais et les groupes migrants plus récents. Pourtant, on sait que les Canadiens français, qui étaient prêts à recevoir des salaires moins élevés, ont suscité un fort ressentiment de la part des autres travailleurs des industries de la Nouvelle-Angleterre. À Fall River, les knobsticks (scabs) sont identifiés d'abord aux briseurs de grève canadiens-français, ce qui leur vaut d'être " mis au ban de la société

\footnotetext{
${ }^{48}$ Nevers, L'âme américaine, tome II, p. 176.

${ }^{49}$ Timothy J. Meagher, Inventing Irish America: Generation, Class, and Ethnic Identity in a New England City, 1880-1928, Notre Dame (IN), University of Notre Dame Press, 2001, p. 202.
} 
par tout le monde ${ }^{50}$ ». Par ailleurs, la résurgence de l'American Protective Association dans les années 1890 annonçait la recrudescence de campagnes contre les étrangers, qui rappelaient les exactions, un demi-siècle plus tôt, des Know-Nothing ${ }^{51}$. "It was these changes", résume un historien, " - the collapse of old arrangements that had smothered ethnic tension and blurred ethnic boundaries and linked the Irish to members of the Yankee elite, and the eruption of seething, turbulent ethnic rivalry and competition - that doomed the old accommodation. And it was these changes, too, that spurred an ethnocentric revival among the Irish ${ }^{52}$. "Nevers écartait ce genre d'explication à la fois économique, sociale et politique. Fidèle à la science des races, il préférait citer une autre liste de motifs, cette fois tirés d'une psychologie des peuples assez sommaire.

Il y avait d'abord le caractère même de l'Irlandais, reflet d'une existence menée au milieu d'une nature sauvage et revêche. Nevers citait les paroles très dures d'un journaliste irlandais : " [...] nous sommes une horde de sauvages avilis et manquant de la gratitude, des instincts de noblesse qui caractérisent l'enfant des bois ${ }^{53}$. " Le Celte, comme nous l'avons vu plus haut, n'aurait rien perdu de sa personnalité nerveuse en traversant l'océan : "Le Celte qui domine absolument, aujourd'hui, dans l'Union est essentiellement et avant tout, un combatif qui lutterait rien que pour le plaisir de la lutte, rien que pour l'ivresse de la bataille ${ }^{54}$." Ce qu'il aime, ce sont les " effets puissants et rapides ". "Il confond le volume avec la grandeur ${ }^{55}$."

${ }^{50}$ Ralph D. Vicero, Immigration of French Canadians to New England, 1840-1900: A Geographical Analysis, thèse de doctorat, Université du Wisconsin, 1968, p. 350.

${ }^{51}$ Dans les années 1840 et 1850 , les Know-Nothing constituaient un parti politique xénophobe et antipapiste qui visait tout particulièrement les immigrants irlandais. Une tendance similaire se remarque au Canada (David A. Wilson (dir.), The Orange Order in Canada, Dublin, Four Courts Press, 2007).

${ }_{52}$ Meagher, Inventing Irish America, p. 207. Lire également, sur cette période, Kevin Kenny, The American Irish: A History, New York, Longman, 2000, p. 171-179.

${ }^{53}$ Cité dans Nevers, L'àme américaine, tome II, p. 180-181.

${ }^{54}$ Ibid., p. 312.

${ }^{55}$ James Bryce, cité dans Ibid., p. 58. 
À côté de cette hypothèse atavique, Nevers formulait une explication morale. La morgue, voire la haine des «nativistes" (nom sous lequel on désignait les partisans de l'assimilation des immigrants à la culture anglo-saxonne) auraient fait des ravages dans les rangs des Irlandais au milieu du XIx ${ }^{e}$ siècle, eux dont l'accent était raillé, dont la pauvreté était dénigrée et qui furent sans cesse bafoués comme papistes $^{56}$. Le mépris dont ces derniers furent victimes, au travail, devant les tribunaux, à l'école, dans la rue, finissait, disait Nevers, par miner leur sens moral. Le dénigrement qui les accueillait à leur arrivée en sol américain avait corrompu leurs cœurs et leurs esprits, leur enlevant cette dignité qui rend les âmes élevées tolérantes et magnanimes - sauf, justement, chez les classes plus aisées, qui avaient, elles, su conserver intact l'amour de la patrie perdue. C'est ainsi que Nevers refusait de considérer les stratégies de réussite des immigrés irlandais, préférant insister sur la déchéance de leur comportement. Ce n'était pourtant pas un hasard si les "petits blancs", ces individus qui, quoique pauvres, ne pouvaient s'empêcher d'entretenir toutes sortes de préjugés envers ceux situés encore plus bas qu'eux, se seraient recrutés principalement parmi les descendants de gens venus de la verte Érin. Ils avaient l'avantage, face aux Allemands ou aux Espagnols qui baragouinaient l'anglais, de pouvoir, eux, jouer aux Anglais de vieille souche. Ils en profitaient pour $s^{\prime}$ assurer un avantage facile sur les étrangers crédules qui s'établissaient dans les campagnes et les villes. Ils n'étaient peut-être pas presbytériens ou baptistes, mais ils arboraient au moins cette marque de distinction de parler couramment la langue du commerce et des affaires. En grossissant cet avantage, qu'ils savaient toutefois fragile, ils pouvaient accroître la distance qui les séparait des autres émigrés, peut-être mieux nantis qu'eux, peut-être plus éduqués qu'eux, mais pas aussi Américains qu'eux, pour autant bien sûr qu'une langue différente fut un critère d'hyphenatedness. Il est curieux que Nevers n'ait pas compris qu'il était naturel

${ }^{56}$ David H. Bennett, The Party of Fear: The American Far Right from Nativism to Militia Movement, New York, Vintage Books, 1995. 
pour eux de se faire les champions du mouvement " un drapeau, une langue " de manière à désamorcer la sempiternelle accusation de lèse-république, alors que de nombreux passages de L'âme américaine évoquent la plus-value associée à la maîtrise de la langue de Shakespeare.

Une dernière motivation qui se profilait derrière le comportement agressif des Irlandais découlait des tribulations de leur histoire coloniale. "Il semble, confiait Nevers, qu'il y ait des défauts et des vices spéciaux pour les vaincus et les opprimés. L'homme qui ne se meut pas et ne respire pas librement est bientôt atteint de certaines maladies internes, il s'ankylose et se déforme; il en est pour les peuples comme pour les individus ${ }^{57}$. " C'était le cas du peuple irlandais, trop longtemps tenu dans les chaînes de la couronne britannique. À force de subir vexations et exploitation, ce peuple fier avait fini par intérioriser la nécessité du rapport entre le maître et l'esclave. Nevers allait jusqu'à écrire qu'il avait gardé en Amérique "la nostalgie de l'oppression ${ }^{58}$ ». Il avait besoin de faire souffrir les autres pour la bonne raison qu'il avait souffert lui-même. Ayant subi des siècles de domination et d'exploitation, il ne savait être que victime ou bourreau. L'oppression serait "passée dans leurs habitudes; si on ne l'exerçait pas contre eux, ils trouvaient naturel de l'exercer contre les autres". "L'ancien opprimé se redresse, le vaincu d'autrefois devient un dominateur; les qualités et les défauts qui s'étaient développés en lui dans la servitude survivent dans la liberté, ils s'exagèrent souvent ${ }^{59}$. " Nevers formulait des réflexions semblables en ce qui concerne les prêtres irlandais. Indifférent aux suppliques envoyées à Rome qui le pressaient de modérer ses ardeurs assimilatrices, le clergé irlandais " paraît avoir la nostalgie de l'oppression; l'oppression qui pendant si longtemps, au pays natal, a été l'un des éléments de sa vie, fait désormais partie de son hérédité; si on ne le tyrannise pas, il sent le

\footnotetext{
${ }^{57}$ Nevers, L'âme américaine, tome II, p. 57.

${ }^{58}$ Ibid., p. 59.

${ }^{59}$ Ibid., p. 58.
} 
besoin de tyranniser les autres ${ }^{60}$ ». Loin de l'avoir rendu plus sensible aux souffrances, des siècles d'intolérance l'auraient pénétré d'un ressentiment terrible et hargneux.

Il aurait fallu pouvoir faire entendre raison à un tel individu à qui avait été transmise, comme une maladie, la rage de la persécution. Nevers doutait néanmoins que des hommes affligés de ces " tares ineffaçables " puissent jamais comprendre l'odieux de leurs actions, tellement les aveuglait une longue histoire de privations et de dégradations.

\section{Mépris des autres et politiques assimilatrices}

Nevers refusait, sinon seulement au passage, de citer des motifs très concrets pour comprendre la conversion des immigrants américains à l'anglais. Pourquoi donc le gros des immigrés aurait-il fait le sacrifice d'alourdir sa marche vers l'Ouest de mille souvenirs devenus inutiles, alors que l'attendaient toutes les promesses matérielles du Nouveau Monde? Pour quelles raisons ces hommes et ces femmes, qui avaient quitté leur pays pour échapper à la tyrannie, à la désolation et à l'injustice, auraient juré fidélité à des traditions qui semblaient les condamner à vivre dans ces quartiers miséreux qui avaient pour noms Petite Italie ou Chinatown? En outre, l'école, le travail et l'Église élevaient quantité de petits et de grands obstacles à la conservation des langues des immigrants. Les Canadiens français se fondirent dans la société dominante d'abord parce qu'ils concevaient cette stratégie comme beaucoup plus gagnante. «Étant d'origine française, j'aurais dû apprendre deux langues, confiait un témoin. Toutefois, mes parents croyaient que si je n'apprenais qu'une langue je réussirais mieux qu'en allant à une école bilingue ${ }^{61}$. „ Il y avait là un pragmatisme naturel

${ }^{60}$ Ibid., p. 176.

${ }^{61}$ "Being of French descent, I would have had to learn two languages; and my parents felt that if I learned one language, I'd end up better off than going to a school with two languages " (Témoignage de Raymond Dubois (nom fictif), dans Tamara K. Hareven et Randolph Langenbach (dir.), Amoskeag: Life and Work in an American Factory-City, New York, Pantheon Books, 1978, p. 164). 
pour ceux et celles qui cherchaient à hausser leur niveau de vie dans la société nouvelle. Sacrifiant ce qu'on appelait les quatre piliers de la survivance, ils se joignaient aux paroisses irlandaises, envoyaient leurs enfants à l'école publique (qui était gratuite et dont l'année scolaire était plus courte, ce qui permettait aux enfants de travailler plus longtemps à l'usine), joignaient des sociétés mutuelles de langue anglaise et s'abonnaient aux journaux américains ${ }^{62}$. Ils pratiquaient aussi fréquemment l'exogamie ${ }^{63}$. Toutefois, Nevers refusait de considérer les trajectoires individuelles de cette façon et préférait toujours revenir aux manifestations d'intolérance de la part des nativistes pour comprendre l'abandon de la langue maternelle et des traditions nationales.

Son aveuglement face au pragmatisme adopté par l'épiscopat romain aux États-Unis est semblable. Les pages de L'âme américaine décrivent les machinations et les stratégies ourdies par les Irlandais pour imposer des curés et des évêques de leur race, même là où la population canadienne-française réclamait avec force des pasteurs appartenant à sa nationalité. "Le zèle assimilateur ou plutôt "anglicisateur" de l'épiscopat irlandais constitue, à l'heure qu'il est, le grand obstacle aux progrès du catholicisme dans l'Union américaine $e^{64}$. " Il faut dire que, parmi une kyrielle de vexations, les élites francoaméricaines avaient été échauffées par "l'affaire de Danielson " (18941897), qui faisait suite à "l'affaire de la Flint " (1884-1886), affaires provoquées dans un cas comme dans l'autre par le refus des évêques irlandais de nommer des curés canadiens dans des paroisses majo-

${ }^{62}$ James Bryce fait une analyse lucide du processus par lequel les journaux, l'usine et l'école agissent comme des solvants des identités nationales des immigrants (The American Commonwealth, p. 358-359).

${ }^{63}$ Roby, Les Franco-Américains de la Nouvelle-Angleterre, p. 100.

${ }^{64}$ Nevers, L'âme américaine, tome II, p. 176. L'épisode des conflits avec le clergé irlandais a été longuement discuté dans l'historiographie. Pour un bon résumé de la "menace irlandaise ", lire Yves Roby, "Les Franco-Américains et les évêques "irlandais" ", dans Claire Quintal (dir.), Religion catholique et appartenance franco-américaine, Worcester (MA), Institut français, Assumption College, 1993, p. 11-16. 
ritairement francophones ${ }^{65}$. « Dès le tournant du siècle, la curie romaine avait adopté une politique américaine (et canadienne-anglaise) qui préconisait l'assimilation de toute ethnie catholique sous l'égide de l'épiscopat irlandais ${ }^{66}$. $\mathrm{Or}$, quoiqu'elle n'ait point refusé par principe de nommer des prêtres canadiens-français dans les paroisses où ils formaient une majorité décisive ${ }^{67}$, l'administration vaticane penchait vers les Irlandais pour des raisons évidentes : outre l'embarras où elle serait placée si elle tentait de tracer d'illusoires frontières ethniques autour de paroisses bigarrées, elle avait compris que, pour devenir prépondérante en Amérique du Nord, l'Église devait parler la langue de la majorité. Il ne s'agissait pas de conquérir l'Amérique pour défendre quelques langues nationales, mais pour prendre possession des âmes du continent et les rassembler au sein de la religion véritable ${ }^{68}$. En outre, les Irlandais contribuaient plus généreusement aux coffres dégarnis de la papauté, à un moment où celle-ci, dépouillée de ses États, en avait bien besoin. Enfin, les Canadiens français penchaient davantage que les Irlandais vers le Parti républicain, à un moment où le Parti démocrate commençait à dominer la politique fédérale ${ }^{69}$.

${ }^{65}$ Roby, Les Franco-Américains de la Nouvelle-Angleterre.

${ }^{66}$ André Sénécal, "La thèse messianique et les Franco-Américains ", Revue d'histoire de l'Amérique française, vol. XXXIV, $\mathrm{n}^{\circ} 4$ (mars 1981), p. 555.

${ }^{67}$ Edmond de Nevers affirmait que les évêques irlandais avaient toujours refusé d'accorder des prêtres de leur nationalité aux Canadiens français de la NouvelleAngleterre, alors qu'en vérité, quatorze autorisations furent accordées de 1861 à 1870 , vingt-huit de 1871 à 1880 , vingt-trois de 1881 à 1890 , et dix-neuf dans la dernière décennie du siècle (Roby, Les Franco-Américains de la Nouvelle-Angleterre, p. 115, ainsi que p. 125-126). Il demeure que les deux tiers des sièges épiscopaux de la Nouvelle-Angleterre appartenaient à des Irlandais. Les Allemands occupaient un rang moindre, mais honorable ( $15 \%)$ dans la hiérarchie, suivis de très loin par les Polonais, puis, en proportion négligeable, par les Canadiens français et les Italiens (Dolan, The American Catholic Experience, p. 302).

68 "Il y a des gens en Amérique et en Europe qui ont l'impression que les États-Unis sont un vaste Congo qui peut être morcelé en autant de colonies étrangères " ( $\mathrm{M}^{\mathrm{gr}} \mathrm{Ireland}$, cité dans John J. Meng, "Cahenslyism: The Second Chapter, 1891-1900 ", The Catholic Historical Review, vol. XXXII, n³ (octobre 1946), note 114, p. 321).

${ }^{69}$ " [...] the French thus began drifting away from the Celtic-dominated Democrats to the Yankee-led GOP over the latter half of the 1890s " (Ronald A. Petrin, French 
Cela faisait assez de mobiles pour ne pas avoir à invoquer un quelconque instinct de domination des Irlandais. Mais Nevers, comme un bon nombre de ses compatriotes déçus par les échecs répétés d'obtenir des prêtres parlant leur langue, préférait deviner les travers de la psychologie du peuple hibernien dans ces agissements hostiles. Il n'hésitait pas à " diaboliser ${ }^{70}$ » le clergé irlandais, allant jusqu’à craindre que "l'avenir de l'Église catholique aux États-Unis " ne soit "sacrifié au rêve d'anglicisation d'un certain nombre d'évêques, [et] à la satisfaction de leurs instincts tyranniques ${ }^{71}$ ». Selon lui, il n'était pas dans l'intérêt de l'Église de laisser perdurer une situation aussi inique et néfaste au rayonnement romain dans la mesure où il croyait, comme Henri Bourassa et l'ensemble des partisans du cahenslyisme, que la langue était gardienne de la foi ${ }^{72}$. La crise sentinelliste qui agita la communauté

Canadians in Massachusetts Politics, 1885-1915: Ethnicity and Political Pragmatism, Philadelphia, The Balch Institute Press, 1990, p. 75-94).

${ }^{70}$ Roby, Les Franco-Américains de la Nouvelle-Angleterre, p. 154.

${ }^{71}$ Nevers, L'âme américaine, tome II, p. 183. Il faut dire que des commentateurs, convaincus de la " manifest destiny" des catholiques aux États-Unis, ne se gênaient pas pour alimenter les peurs des nationalistes canadiens-français. "L'Irlande fut soumise, déclarait John Joseph Lynch en 1860, afin d'incorporer plus efficacement ses habitants à la nation anglaise... Ceux-ci résistèrent mais on les contraignit par la force à apprendre l'anglais. Ce sont là les desseins de Dieu. Fouettés à l'école parce qu'ils ne savaient pas leurs leçons d'anglais, les enfants irlandais ne soupçonnaient pas que Dieu destinait la langue anglaise à répandre par leur bouche la parole de son Divin Fils dans le vaste monde " (Cité dans Roberto Perin, Rome et le Canada : la bureaucratie vaticane et la question nationale, 1870-1903, Montréal, Boréal, 1993, p. 26).

${ }^{72}$ Le cahenslyisme, du nom d'un de ses principaux protagonistes, Peter Paul Cahensly, réclamait davantage d'autonomie ecclésiastique pour les groupes germanophones et les autres communautés allophones des États-Unis, ce qui se serait traduit par l'élection d'un plus grand nombre d'évêques " nationaux ». Aux " américanistes", qui prétendaient qu'il s'agissait d'un complot ourdi pour germaniser le continent, les " européanistes » répondaient que c'était la seule voie pour empêcher les immigrants de tomber dans le paganisme et l'athéisme (Meng, "Cahenslyisme: The Second Chapter, 1891-1910 ", p. 302-340). Le slogan de ces derniers - Allemands, Polonais, Italiens - était, comme au Canada français, "Language Saves the Faith " (Dolan, The American Catholic Experience, p. 297). 
canadienne-française un quart de siècle plus tard allait montrer à quel point Nevers se trompait sur la realpolitik suivie par le Vatican ${ }^{73}$.

Nevers considérait que l'instrument ultime du melting pot américain relevait davantage du cœur que de la raison. Le véritable coupable de l'apostasie nationale, c'était le mépris quotidien, distillé de manière sourde à travers les journaux et les discours publics, dont on accablait quiconque ne se conformait pas au modèle américanoirlandais. La simple conviction que tout était bigger and better aux États-Unis et que, réciproquement, tout ce qui n'était pas américain était inférieur finissait, au dire de Nevers, par corrompre les âmes les plus hautes. Un racisme des plus plats et des plus stupides avait fini par corrompre l'instinct de survie des immigrés. Plutôt que de supporter les railleries et les moqueries qui faisaient de leur origine une tare, un stigmate honteux, ils préféraient effacer cette infamie en se fondant dans la foule.

Pour éviter que de telles abjurations se multiplient à l'avenir, Nevers a formulé une défense passionnée de sa nationalité. À un moment où ses compatriotes formaient, avec les Italiens et les Européens de l'Est, la deuxième vague d'immigration massive aux États-Unis (après les Anglais, les Allemands, les Scandinaves et les Irlandais), Nevers n'a cessé de vanter les qualités et la grandeur de ces travailleurs pauvres et analphabètes ${ }^{74}$. Par ses paroles et des écrits, il cherchait à contrecarrer l'opinion répandue parmi le public américain selon laquelle les Canadiens français seraient « ignorants,

${ }^{73}$ Parmi une abondante littérature sur la crise sentinelliste, lire Richard S. Sorrell, The Sentinelle Affair (1924-1929) and militant "survivance": The Franco-American Experience in Woonsocket, Rhode Island, thèse de doctorat, State University of New York at Buffalo, 1975; Roby, Les Franco-Américains de la Nouvelle-Angleterre, p. 273330; Pierre Anctil, Aspects of Class Ideology in a New England Ethnic Minority: The Franco-Americans of Woonsocket, Rhode Island (1865-1929), thèse de doctorat, New School for Social Research, 1980, p. 148-278; Hélène Forget, L'agitation sentinelliste au Rhode Island (1924-1929), mémoire de maîtrise, Université de Montréal, 1953; Damien-Claude Bélanger, "L'abbé Lionel Groulx et la crise sentinelliste ", Mens, vol. I, n 1 (automne 2000), p. 7-36.

${ }^{74}$ François Weil, Les Franco-Américains, 1860-1980, Paris, Belin, 1989, p. 72. 
immoraux, bien pires que les Chinois de la Californie et un fléau pour la sociétét 75 ". Nevers voulait apprendre à la jeune génération à s'enorgueillir davantage de ses origines pour ne pas baisser la garde devant les assauts des anglophiles. Le génie français lui paraissait sans bornes. Le XIX ${ }^{e}$ siècle, surtout, avait consacré la France au faîte du monde civilisé, et elle y trônait sans égal. Pour l'élégance, elle n'avait pas son pareil; dans le domaine de la science, elle avait fait plus pour le progrès que toute l'Europe réunie; dans le domaine des beaux-arts, elle continuait d'attirer les artistes, qui venaient des quatre coins du monde se former à Paris; dans le domaine littéraire, sa suprématie était, s'il se pouvait, encore plus éclatante. Comment donc ne pas vibrer de fierté à entendre parler « la langue qu'ont illustrée tant d'hommes de génie, qu'a policée à travers les siècles, la société la plus élégante et la plus raffinée de l'Europe, qui est encore aujourd'hui celle de la diplomatie et des chancelleries, la langue dont la connaissance constitue un brevet de distinction pour les aristocraties du monde entier ${ }^{76}$ "? Comment vouloir dénier le droit aux descendants d'un peuple aussi illustre de prospérer en terre américaine? Nevers reprenait ici un discours grandement partagé par les Canadiens français de son époque, pour qui les Anglo-Américains seraient des gens matérialistes, égoïstes et grossiers, alors que les races latines seraient, elles, moralement et intellectuellement supérieures ${ }^{77}$.

Cependant, que la langue soit belle ou laide, qu'elle soit aristocratique ou roturière, elle possède pour Nevers des droits sacrés que nul ne saurait enfreindre sans impunité. C'est elle que l'enfant apprend sur les genoux de sa mère, qu'il assimile avec reconnaissance et tendresse dès son plus jeune âge. Dans un texte maintes fois reproduit dans les journaux et tiré de L'avenir du peuple canadien-français,

${ }^{75}$ Dennis R. Garff, Heirs of New France: An Ethnic Minority in Search of Security, thèse de doctorat, Tufts University, Fletcher School of Law and Diplomacy, 1970, note 113 , p. 118 .

${ }^{76}$ Nevers, "Encore quelques mots sur les Anglais et nous ", p. 1.

${ }^{77}$ Lire, parmi une ribambelle d'exemples, l'abbé Henri-Raymond Casgrain, Euvres complètes, vol. I, Montréal, Beauchemin, 1896, p. 369. 
Nevers insistait sur le lien intime et vital qui unit l'âme d'une personne à sa langue maternelle. "Oh! la langue maternelle, génie familier, qui s'est introduit, infiltré peu à peu dans nos âmes, avec les premiers balbutiements, langue des aïeux, combien elle l'emporte sur tout autre idiome, acquis depuis, à l'âge d'homme, ou même d'adolescent, de collégien ${ }^{78}$ ! A Au-delà de la langue, c'est l'ensemble de la culture d'un peuple qui devait être préservé des contaminations extérieures afin de conserver santé et vigueur. Ceux qui demeuraient fidèles à la mémoire de la patrie perdue étaient en effet sans cesse vivifiés par ce souvenir. Loin de les abaisser en les tirant vers leurs racines, ce sentiment de fierté naturelle les élevait au dessus des masses assimilées ${ }^{79}$.

À l'opposé, renier les valeurs de sa nationalité, c'était se couper de soi-même, et éventuellement dégénérer. Les individus qui acceptaient de s'assimiler à l'élément dominant s'abâtardissaient et finissaient pas croupir dans une médiocrité coupable. Le renégat de sa race portait la haine de soi en lui comme un feu dévorant. Dans l'abandon du passé et le reniement des traditions, Nevers lisait les signes avant-coureurs d'une terrible décadence, décadence qui allait se conclure par l'envahissement progressif d'un esprit égoïste, tapageur et intéressé. Or, justement, pour Nevers, ainsi en était-il des nombreux Irlandais qui avaient accepté en émigrant de rompre le courant qui les unissait à leurs ancêtres. «Souvenirs enfouis, affections étouffées, aptitudes héréditaires éteintes. Voilà ce qui se trouve à la base de l'arbre familial de presque tous les descendants d'émigrés $[. .$.$] celtes$ aux États-Unis. L'arbre n'a pas dû en être fécondé ${ }^{80}$. " La bouche de ces Irlandais se remplissait de slogans patriotiques qui célébraient les États-Unis comme le plus grand, le plus beau et le meilleur pays du monde afin de cacher par cette surenchère de chauvinisme la honte du pays natal. Une telle abdication annonçait des jours sombres pour

\footnotetext{
${ }^{78}$ Nevers, L'avenir du peuple canadien-français, p. 127.

${ }^{79}$ Nevers, L'âme américaine, tome II, p. 64.

${ }^{80}$ Ibid., p. 57.
} 
les États-Unis. Les faillites répétées et les taux mirobolants de divorce ne sont que quelques-uns des exemples, cités par Nevers, qui illustraient une corruption morale croissante. Ainsi, la surexcitation nerveuse qui affectait le tempérament américain viendrait de l'influence celtique. "La vapeur, l'électricité réalisent des prodiges. Tout est bruit, confusion, agitation. C'est le règne du journaliste, le règne $\mathrm{du}$ politicien, le règne de la liberté absolue et sans entraves, et c'est aussi le triomphe de Paddy ['Irlandais], qu'il se dissimule ou non sous la peau de Jonathan [l'Américain] ${ }^{81}$. "Sous l'influence délétère des Irlandais, les habitants des États-Unis souffraient désormais d'une "nervosité excessive ", de "maladies à base de névrose", qui provoquaient fièvre et agitation. Plus que d'autres peuples, ils auraient été affligés de myopie, de calvitie, de dyspepsie, de migraine, de rhumatisme!

Nevers ne désespérait guère de l'avenir de son peuple en Amérique, même si l'heure était grave. Il ne pouvait croire que les Canadiens français subiraient le sort de ces immigrés plus pusillanimes qui n'avaient su résister au rejet dont ils étaient victimes et avaient passé par millions "sous les fourches caudines du mépris anglo-irlandosaxon ". Au tournant du Xxe siècle, par exemple, seuls quelques îlots de populations germanophones résistaient dans l'Ouest et cherchaient tant bien que mal à perpétuer les vieilles traditions, là où, cinq ans plus tôt, Nevers avait cru distinguer des communautés culturelles florissantes. Le reste des immigrants avait rapidement abandonné l'idée de vivre en communion de pensée après avoir laissé ses leaders s'époumoner lors de conventions qui se terminaient par d'héroïques professions de foi envers la langue maternelle et les valeurs du passé. Même s'il était conscient que la nation allemande de l'Ouest avait disparu sous les attaques des " anglomaniaques » et que le rêve d'une Petite Allemagne s'était écroulé, Nevers ne pouvait accepter que les Canadiens français s'assimilent eux aussi sans livrer une chaude bataille. La décadence de la langue française en Louisiane, soumise

${ }^{81}$ Ibid., p. 162. 
à une panoplie d'interdits, ne lui arrachait guère d'inquiétude. Même s'il faisait preuve d'un peu moins de triomphalisme, il conservait en 1900 la foi qui, dans les dernières pages de L'avenir du peuple canadienfrançais, traçait pour sa patrie une destinée glorieuse. Les remarques finales de L'âme américaine ne laissent pas d'être optimistes. Les Canadiens français allaient survivre au fanatisme des " pansaxonnistes". Les idéaux d'ouverture et de respect des États-Unis, "produits supérieurs de [la] saine hérédité » des pères fondateurs, finiraient par prévaloir contre les réflexes de race des descendants des Celtes qui peuplaient désormais en masse le continent. La tension entre la mesure des pionniers et le fanatisme des émigrants irlandais serait finalement résolue en faveur des premiers. Edmond de Nevers ne pouvait croire, il ne voulait croire que le lumineux exemple de probité et d'humanité de la République américaine se perde dans les sombres dispositions des Irlandais.

\section{Conclusion}

Que penser en conclusion des théories raciales de Nevers? Que dire de son analyse des atavismes irlandais? Après une certaine vogue qui perdurera jusqu'après sa mort ${ }^{82}$, ses idées sont, bien entendu, tout à fait discréditées aujourd'hui, nul chercheur actuel ne continuant à croire à l'exercice d'une consanguinité sadique ou masochiste pour expliquer le comportement assimilateur des Irlandais établis aux États-Unis. Le mépris irrédentiste, "l'exclusivisme intransigeant et dédaigneux " qui distinguerait ces derniers au sein des autres nations qui peuplent l'Occident sont à mettre au compte des chimères idéologiques de la fin du XIX ${ }^{e}$ siècle.

Or, l'explication mi-historique, mi-génétique de Nevers paraît d'autant plus douteuse qu'en d'autres passages de son œuvre l'auteur postule que l'oppression serait affaire de cycle. Une nation pourrait

${ }^{82}$ Catherine Pomeyrols, Les intellectuels québécois : formation et engagements 1919-1939, Paris et Montréal, L'Harmattan, 1996. 
être portée à mépriser les autres peuples parce qu'elle aurait pris l'habitude d'imposer par la force ses mœurs et ses institutions aux groupements plus faibles, ce qui semblait être le cas, pour Nevers, de l'Angleterre, tout comme une nation pourrait succomber à des inclinaisons persécutrices parce qu'elle aurait longtemps subi un joug étranger, comme l'exemple irlandais semblait le prouver. Mais une nation pouvait aussi se retrouver dans le rôle de l'oppresseur parce qu'elle n'avait jamais combattu de manière violente des étrangers une constatation que le lecteur contemporain pourra trouver assez curieuse. Ainsi, ce serait parce que l'Allemagne n'avait pas connu au Moyen Âge des massacres semblables à ceux des contrées voisines qu'elle aurait éprouvé le besoin de tyranniser les Polonais au XIX $X^{e}$ siècle. "Est-il donc écrit dans le livre des destinées que chaque nation devra, à son tour, pratiquer l'oppression et la tyrannie? Alors que la France expulsait les Huguenots, que l'Espagne allumait des bûchers, que l'Angleterre martyrisait l'Irlande, l'Allemagne n'eut ni inquisition, ni autodafés, ni édits de proscription; elle prend sa revanche ${ }^{83}$. " Il semble donc que, pour Nevers, n'importe quelle raison ait été bonne pour expliquer la propension d'un groupe à en terroriser un autre, ce qui enlève toute pertinence à son schème d'analyse. Peu importe la situation, Nevers finissait toujours par invoquer une clef interprétative globale : un groupe opprimait parce qu'il avait toujours opprimé, parce qu'il n'avait jamais été opprimé ou parce qu'il avait toujours été opprimé.

Quand il se faisait sociologue, Nevers touchait plus juste, et son hypothèse d'une volonté de différenciation de la part des Irlandais face à un groupe, les Canadiens français, plus humble que lui, au moins par le handicap de la langue, s'avère fort plausible. Pour s'assurer un avantage sur les autres immigrants, les Irlandais avaient besoin de consacrer l'Amérique comme une terre anglaise. À chacun sa défense : des Allemands pouvaient se targuer d'être protestants, alors que les Irlandais insistaient sur le fait qu'ils parlaient anglais; quant aux

${ }^{83}$ Nevers, L'âme américaine, tome II, p. 194. 
Canadiens français, qui ne pouvaient ni l'un ni l'autre, ils se vantaient d'être d'honnêtes travailleurs et des citoyens paisibles. Mais, pour maints politiciens, la vaillance au travail et la douceur des mœurs ne suffisaient pas à assurer l'intégration dans la société américaine, comme l'auraient prouvé, de l'avis de plusieurs auteurs, l'exemple des Chinois de la Californie qui s'isolaient dans leurs quartiers et refusaient de participer aux institutions de la société plus large. Il aurait fallu cependant creuser davantage ce sillon, mesurer le poids des réseaux sociaux dans la conservation des cultures, analyser la rationalité des acteurs en quête de travail, faire œuvre d'ethnologue dans l'interprétation des comportements des groupes. Trop pressé d'arriver à ses conclusions patriotiques, Nevers semblait refuser de lire dans le problème de l'assimilation autre chose qu'une décision morale. Ce faisant, certes, il pouvait se concentrer sur une défense passionnée de la langue française, mais il s'empêchait aussi de reconnaître que la simple fierté de race s'avérait en définitive de peu d'efficacité pour renverser les tendances à l'assimilation des Canadiens français. Nulle part dans ses écrits Nevers n'a-t-il relevé les nombreux obstacles qui se dressaient devant ceux et celles qui voulaient transmettre leur langue maternelle à leurs enfants. Il ne concevait pas que l'abandon des traditions puisse découler, au grand dam des élites nationalistes, d'une volonté d'adaptation des enfants des immigrés à la société américaine ${ }^{84}$. À l'en croire, les immigrants abandonnaient la langue de leurs ancêtres uniquement par manque de fierté de race. Il pouvait par conséquent placer la solution à l'apostasie nationale à la même hauteur que le problème du mépris infligé par les Irlandais à ceux qui ne parlaient pas anglais. Il fallait combattre le feu par le feu : la fierté par la fierté. C'est ainsi que les Irlandais, de curieuse façon, montraient l'exemple. Ils étaient le modèle de ce que les Canadiens français devaient devenir s'ils voulaient survivre et prospérer dans

${ }^{84}$ C'est la thèse que développe Yves Roby dans « La paroisse franco-américaine (18501976) ", dans Serge Courville et Normand Séguin (dir.), Atlas historique du Québec : la paroisse, Québec, Les Presses de l'Université Laval, 2001, p. 251-262. 
un environnement hostile : durs, indomptables, condescendants et fiers.

Ce n'est pas seulement sur ce point que sa défense passionnée du droit à l'existence de son peuple ne faisait que reprendre, dans une traduction inverse, les arguments de ses adversaires. Nevers prêchait l'égalité des races contre les partisans de la suprématie de la civilisation anglo-saxonne, mais sombrait trop souvent dans un panégyrique de la culture française qui reléguait les autres nations à un rang moins honorable. Les Américains avaient beau avoir reçu en partage, selon lui, le génie du commerce, Nevers était convaincu que ce talent ne valait pas celui des arts, de la littérature et de la science, et que l'esprit français valait donc mieux que l'aptitude roturière à brasser des affaires. Plus encore, sa volonté d'œuvrer à l'édification d'une société américaine tolérante et ouverte se heurtait presque immédiatement à son souhait de conserver des frontières ethniques étanches entre les groupes qui constituaient les États-Unis. Nevers ne pouvait concevoir l'idée d'un métissage entre les peuples, sinon sur le mode du reniement et de la décadence. Il proposait jusqu'au rapatriement des Noirs vers l'Afrique afin de contribuer à l'homogénéisation d'une Amérique qu'il jugeait trop diversifiée. Chaque groupe devait s'assembler en fonction de ses caractéristiques propres. Il devenait difficile par conséquent pour Nevers de critiquer les racialistes de son temps qui, convaincus que chaque pays avait besoin de s'unir autour d'une culture, d'une langue et sinon d'une religion communes, prônaient l'assimilation des immigrants, bien qu'il n'ait jamais été jusqu'à revendiquer l'instauration de lois coercitives ou de règlements vexatoires comme ce fut le cas chez quantité de groupes nativistes de l'époque.

D'une part, Nevers donnait raison aux racialistes de chercher à fondre dans la grande fournaise américaine les éléments disparates qui venaient en masse y chercher paix et fortune : pour lui comme pour eux, une nation forte devait d'abord être fondée sur une union morale. D'autre part, Nevers alimentait sans le vouloir leurs craintes face au patriotisme défaillant, sinon schismatique des Canadiens 
français. Nonobstant sa participation aux grandes campagnes de naturalisation à la fin du XIX ${ }^{e}$ siècle, Nevers professait son allégeance première à sa "petite patrie ". Aussi, il fournissait des arguments à ceux qui s'imaginaient que les "Chinese of the Eastern States", comme certains observateurs appelaient les Franco-Américains ${ }^{85}$, représentaient bel et bien des ennemis de l'intérieur qui ne vouaient qu'une fidélité de façade à la République américaine et préféraient vivre à l'écart, entre eux. Ces gens ne donnaient pas plus l'impression de chercher à s'américaniser aux États-Unis qu'ils n'avaient cherché à $s^{\prime}$ angliciser au Canada ${ }^{86}$. Les idées véhiculées par Nevers ne tendaient pas à réfuter mais plutôt à renforcer de telles opinions, confortant le mépris des élites pour ce demi-million de hyphenated Americans qui prétendaient reproduire de Petits Canadas à Suncook, à Southbridge, à Holyoke ou à Nashua. N'avait-on pas quelque raison de craindre ces immigrants qui s'accroissaient à un rythme prodigieux et promettaient de créer bientôt, de la province de Québec à la NouvelleAngleterre, un État français et catholique au sein de l'État républicain ${ }^{87}$ ? N'était-ce point là, justement, le rêve galliciste d'Edmond de Nevers? Malgré ses appels en faveur de la naturalisation, son projet d'édifier en Nouvelle-Angleterre des " citadelles $^{88}$ " de la

${ }^{85}$ Pierre Anctil, " Chinese of the Eastern States, 1881 ", Recherches sociographiques, vol. XXII, n 1 (janvier-avril 1981), p. 125-131. Yves Roby, "L'incident des Chinois des États de l'Est en Nouvelle-Angleterre (1881) et l'identité franco-américaine ", dans Claire Dolan (dir.), Événement, identité et histoire, Sillery, Septentrion, 1991, p. 137-149.

${ }^{86}$ Frances Early, "The Settling - In Process: The Beginnings of Little Canada in Lowell, Massachusetts, in the Late Nineteenth Century ", dans Claire Quintal (dir.), The Little Canadas of New England, French Institute / Assumption College, 1983, p. 2627. Robert Rumilly citait l'édition du 28 décembre 1889 du American, un journal de Boston : "On leur dit qu'en apprenant l'anglais, ils perdront leur langue, leur nationalité, leur religion. On les conserve comme race distincte, soumise au Pape en matière religieuse et politique " (Histoire des Franco-Américains, p. 128).

${ }^{87}$ Garff, Heirs of New France: An Ethnic Minority in Search of Security.

${ }^{88}$ "Au début, nos chefs nous ont exhortés à construire des citadelles en NouvelleAngleterre. Chaque famille, chaque paroisse, chaque école, chaque "Petit Canada" devait se barricader contre l'ennemi qui voulait nous faire du mal " (Gérard-J. Brault, "Les Franco-Américains, la langue française et la construction de l'identité 
nationalité "francoaméricaine ${ }^{89}$ " prêtait flanc à la critique d'unAmericanness que lui réservaient les anglophiles irlandais. Ceux-ci soulignaient, par exemple, qu'en 1900, seulement le tiers des Canadiens français du Massachusetts s'étaient enregistrés comme électeurs ${ }^{90}$. À force de prêcher l'isolement, il était à craindre que l'on en vienne à accuser les Canadiens français de ne savoir vivre que dans des ghettos culturels. En bref, il y avait un danger, auquel Nevers n'échappe pas, d'insister un peu trop fortement sur les caractéristiques raciales des groupes et la nécessaire homogénéité des cultures : celui d'élever des barrières infranchissables entre les communautés qui peuplaient l'Amérique, au moment même où Nevers réclamait le droit des Canadiens français de participer pleinement à la vie sociale et politique des États-Unis.

Dans un compte rendu de L'àme américaine, Ferdinand Brunetière avait sans doute mis en lumière la contradiction principale dans l'argumentaire élaboré par Nevers. Brunetière refusait de considérer l'assimilation des étrangers à la langue anglaise comme un "facteur morbide " contre lequel devaient se révolter les intellectuels. D'une part, il voyait dans l'effort de la République américaine d'incorporer les étrangers une légitime aspiration à former une nation. D'autre part, il croyait que les races étaient destinées à se dissoudre lentement dans le grand brassage des peuples. Il y avait donc là, pour Brunetière, deux tendances opposées : les nations se concentraient de plus en plus autour d'un noyau solide (la langue, bien sûr, mais aussi la culture, les valeurs, les symboles), alors que les races se diluaient progressivement dans les pays de forte immigration comme l'Amérique. D'ailleurs, ajoutait l'auteur français, « la race n'est pas la force mysté-

nationale ", dans Simon Langlois (dir.), Identité et cultures nationales : l'Amérique française en mutation, Québec, Les Presses de l'Université Laval, 1995, p. 81).

${ }^{89}$ Sylvie Baudreau et Yves Frenette, " Historiographie et identité collective en Amérique française : le cas des élites francophones de la Nouvelle-Angleterre, 1872-1991 ", dans Simon Langlois (dir.), Identité et cultures nationales, p. 285.

${ }^{90}$ Petrin, French Canadians in Massachusetts Politics, 1885-1915, p. 75-94. 
rieuse, irréductible et indestructible que l'on dit ${ }^{91}$ \%. Les tempéraments congénitaux lui semblaient bien fragiles pour qu'en une ou deux générations s'effacent les manières d'être et les tournures d'esprit nationales des anciens émigrés. Il n'était pas le type à s'en émouvoir. Quelque chose resterait sans doute de leurs caractères européen, africain ou asiatique, l'Amérique absorbant les éléments hétérogènes pour en nourrir « sa propre substance ». "Il en sera des autres éléments étrangers comme de l'élément irlandais lui-même. S'il est devenu si puissant aux États-Unis, c'est précisément pour n'avoir pas prétendu maintenir son autonomie dans l'Union ${ }^{92}$. " Nevers refusait, bien entendu, d'entendre cet appel. Pour lui, abdiquer sa langue, renier ses traditions, s'assimiler au groupe dominant, embrasser la culture américaine, c'était littéralement perdre son âme, car jamais une âme catholique et française ne pourrait sans se trahir épouser les sensibilités d'une âme anglo-saxonne ou celte. Ce faisant, il entretenait une certaine aporie, réclamant l'inclusion dans l'Union américaine, alors même qu'il cherchait à accentuer les différences, voire les barrières culturelles. Dans le langage de Brunetière, cela revenait à exiger un État-nation diffus et une race homogène dans un siècle qui générait précisément tout le contraire.

\footnotetext{
${ }^{91}$ Brunetière, "L'âme américaine ", p. 694.
}

${ }^{92}$ Ibid., p. 696-697. 